\title{
Controlling the Nucleation and Growth Orientation of Nanocrystalline Carbon Films during Plasma- Assisted Deposition: A Reactive Molecular Dynamics/Monte Carlo Study
}

Di Zhang ${ }^{\dagger}$, Linfa Peng ${ }^{\dagger}$, Xiaobo Li ${ }^{\dagger}$, Peiyun $\mathrm{Yi}^{*,+}$, Xinmin Lai ${ }^{*,+,}$,

'State Key Laboratory of Mechanical System and Vibration, Shanghai Jiao Tong University, Shanghai 200240, P.R. China

${ }^{\ddagger}$ Shanghai Key Laboratory of Digital Manufacture for Thin-walled Structures, Shanghai Jiao Tong University, Shanghai 200240, P.R. China

\section{Corresponding Author}

*E-mail: yipeiyun@sjitu.edu.cn

*E-mail: xmlai@sjtu.edu.cn

\section{Content}

S1. Simulation model details

S2. Time-stamped force-biased Monte Carlo (tfMC) method parameterization results

S3. Graphitization and efficiency tests of six carbon force fields.

S4. Pre-existing stresses induced by different ensembles

S5. XRD calculation details and the definition of crystallinity 


\section{S1. Simulation model details}

More modeling details including the main dimensions used in the deposition and irradiation simulations are shown in Figure S1. In every cycle of the hybrid molecular dynamics and timestamped force-biased Monte Carlo (MD/tfMC) simulations, a carbon atom and several Ar atoms were deposited from the top of the simulation box. The number of Ar atoms was determined based on the experimental data and analyses. Specifically, the real deposition rate of carbon atoms could be calculated from the thickness of the carbon film and the corresponding deposition time. The Ar ion flux density could be estimated by measuring the substrate bias current, which is presented in the main manuscript. Therefore, the actual ratio between the flux densities of carbon atoms and Ar ions was obtained, and then, this ratio was applied to our deposition simulations. In this work, the real deposition rate of carbon atoms was approximately $1.046 \times 10^{19} \mathrm{~m}^{-2} \cdot \mathrm{s}^{-1}$, and the actual Ar ion flux density was approximately $1.0 \times 10^{19}$ to $4.0 \times 10^{19} \mathrm{~m}^{-2} \cdot \mathrm{s}^{-1}$. In the simulations, a wider range of Ar flux densities was used to optimize the plasma environment. Therefore, the C/Ar ratios were finally set at 1 to 10 . All simulated results were visualized with the help of OVITO. ${ }^{1}$ 
(a)

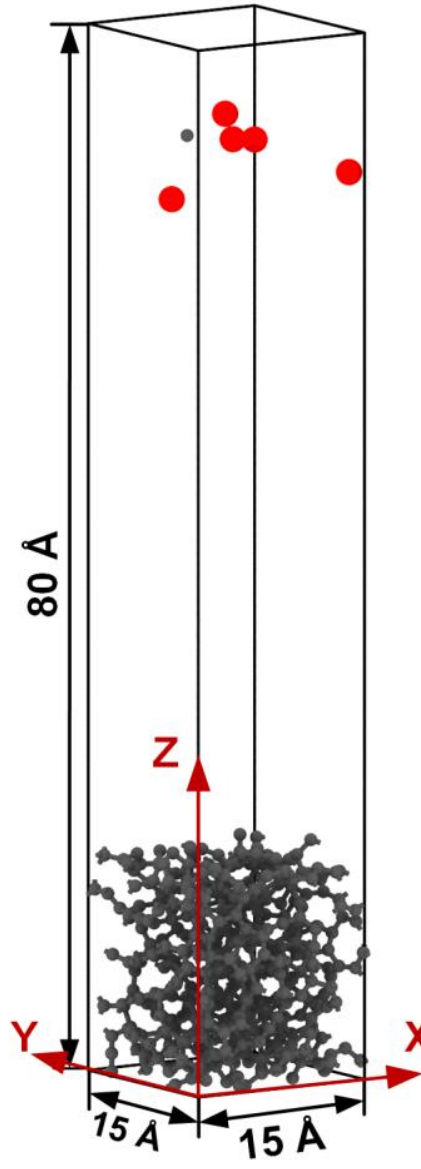

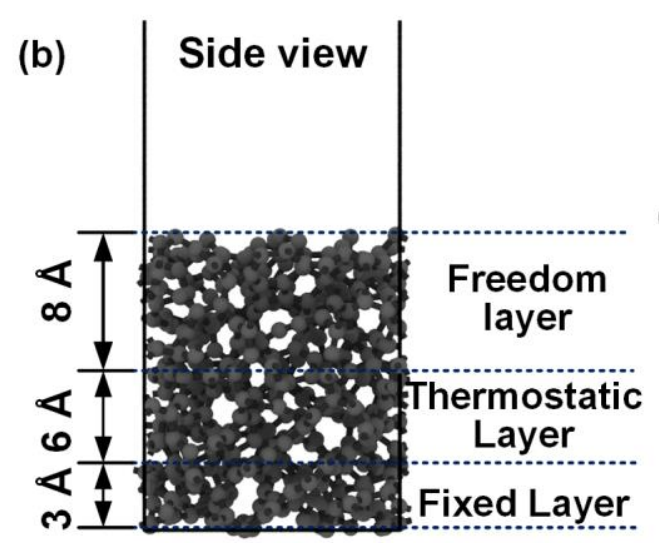
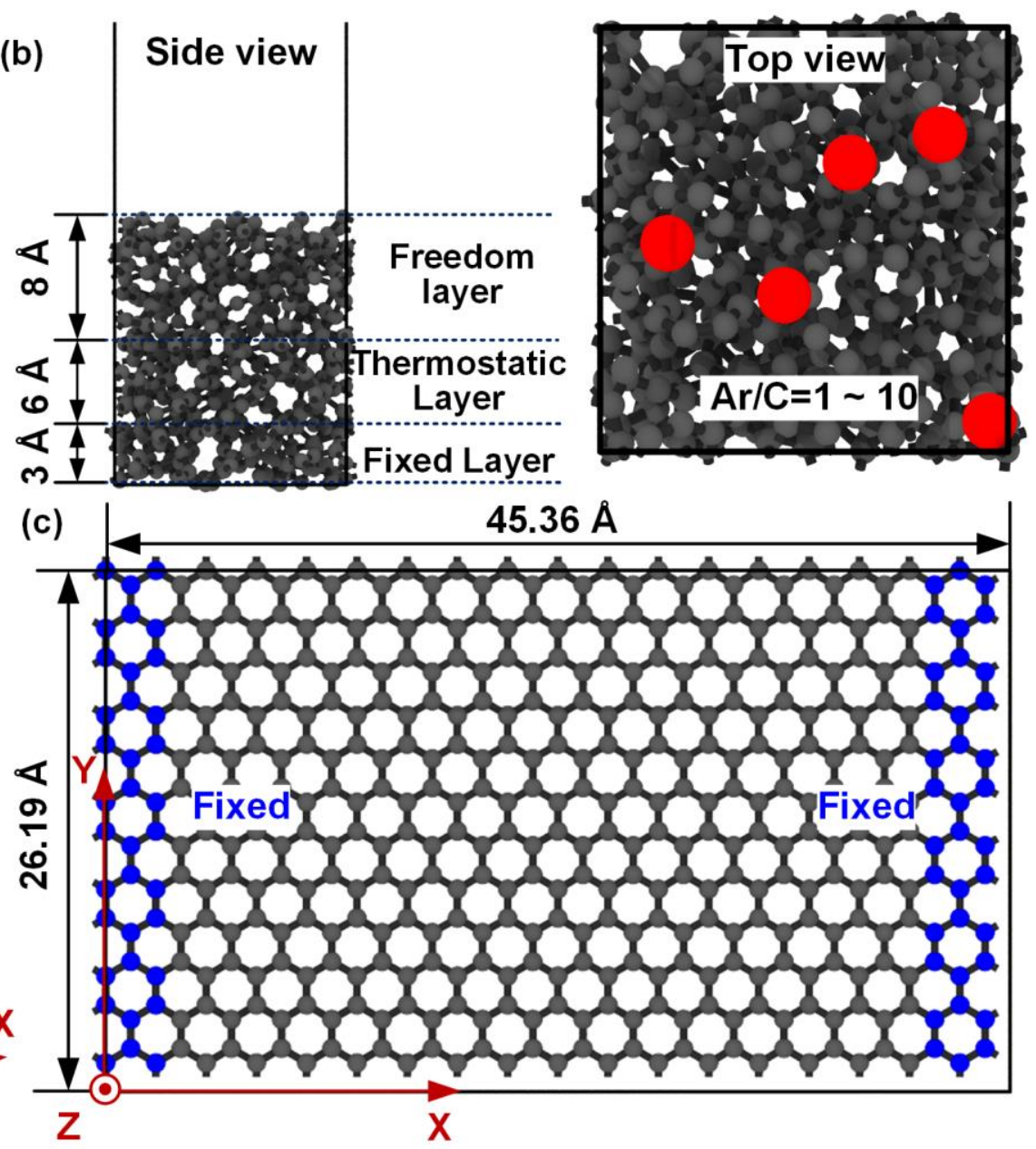

Figure S1. Modeling details of the deposition simulations. (a) The size of the deposition simulation box is $15 \times 15 \times 80 \AA$ and the thickness of the substrate is $17 \AA$. (b) The substrate is divided into three layers, namely, the fixed layer ( $3 \AA$ ) , thermostatic layer $(6 \AA)$, and the freedom layer $(8 \AA$ ). From the top view of the simulation box, the deposited Ar atoms are randomly distributed in the $\mathrm{XY}$ plane. The ratio between the number of $\mathrm{Ar}$ and $\mathrm{C}$ atoms was determined based on the experimental data and analyses. (c) The length and width of the graphene irradiated by Ar ions with different incident angles are $45.36 \AA$ and $26.19 \AA$. The edge areas of graphene were fixed while the middle part of the graphene was not constrained. 


\section{S2. Time-stamped force-biased Monte Carlo (tfMC) method parameterization results}

In the view of a user, two parameters including the temperature $\mathrm{T}$ and maximum allowed displacement $\Delta$ must be determined before the tfMC simulations. The two parameters are the main factors that affect the timescale and physical reality of the tfMC simulations, however, many previous papers that used the tfMC method did not give the specific way to choose them properly. Here, based on what Timonova suggested, ${ }^{2}$ we performed a series of tfMC simulations of a perfect graphite crystal with different $\Delta$ values at different temperatures. For example, at $573 \mathrm{~K}$, different $\Delta$ values from 0.1 to 0.3 were chosen to simulate a perfect graphite crystal, as shown in Figure S2b. For the small $\Delta$ values, the perfect graphite crystal did not show any defects after the hybrid tfMC/MD simulation. However, once the $\Delta$ value was beyond a certain value, for example, 0.21 for $573 \mathrm{~K}$, some defects were observed (at a $\Delta$ value of 0.22 ), and a totally disordered structure was observed at a $\Delta$ value of 0.23 . The same simulation tests were performed in a wide temperature range from $300 \mathrm{~K}$ to $773 \mathrm{~K}$, and the results are presented in Figure S2a. Finally, we set the $\Delta$ value for $773 \mathrm{~K}$ at 0.21 , and the $\Delta$ value for other temperatures was determined by the following relationship ${ }^{3}$ to enable the tfMC simulations with different temperatures to have the same timescale,

$$
t \propto \frac{k_{f} \Delta^{2}}{T}
$$

where $t$ is the timescale of the tfMC step, $k f$ is a force constant and $T$ is the temperature used in the tfMC simulation. 


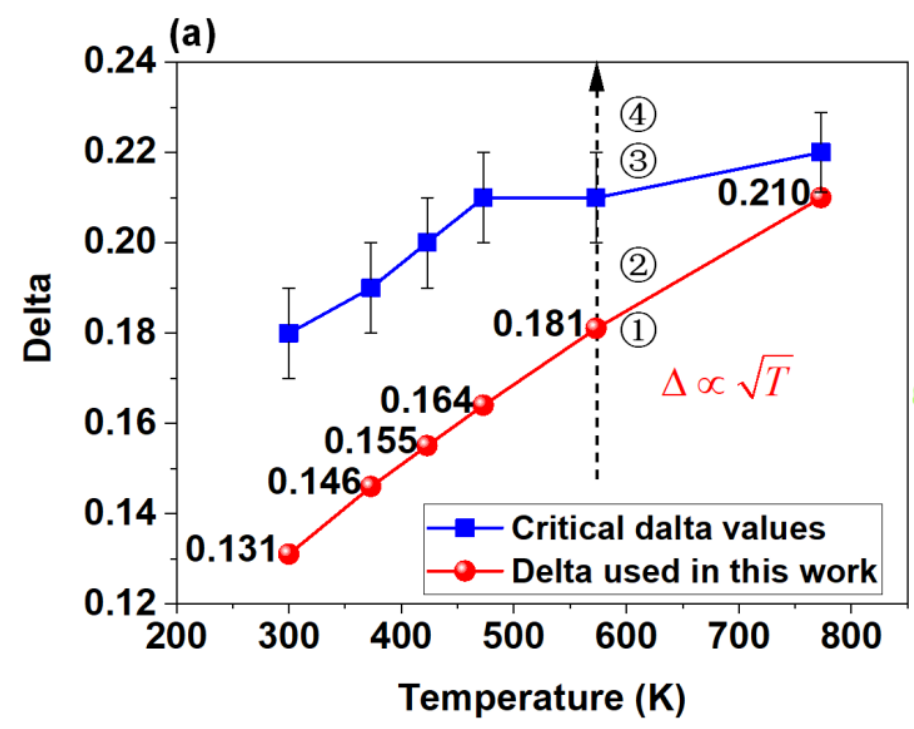

(b)

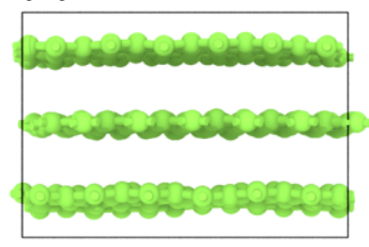

(1) $\Delta=\mathbf{0 . 1 8}$

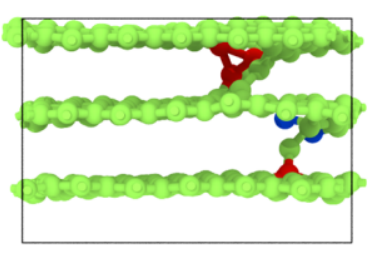

(3) $\Delta=0.22$

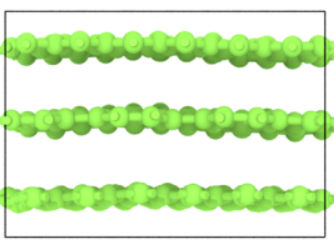

(2) $\Delta=\mathbf{0 . 1 9}$

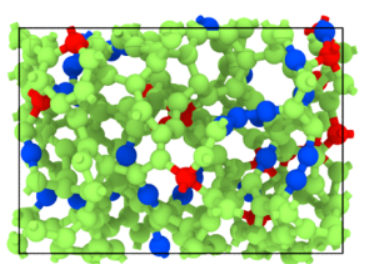

(4) $\Delta=0.23$

Figure S2. Parameterization results of the tfMC methods. (a) Presimulated tests present the critical $\Delta$ values for generating defects in a perfect graphite crystal at different temperatures. Combining the presimulation results with the theoretical relationship between the $\Delta$ value and temperature, the final $\Delta$ values used in this work are also shown in this figure. (b) At $573 \mathrm{~K}$, with the increase in the $\Delta$ value, a perfect graphite crystal is gradually destroyed, and the critical $\Delta$ value is 0.21 for $573 \mathrm{~K}$.

\section{S3. Graphitization and efficiency tests of six carbon force fields}

The interaction between carbon atoms has proved to be difficult to describe due to the complexity of the competing hybridizations and long-range effects associated with $\pi$ electrons. Though carbon potential functions have been developed for more than 30 years since the first potential was released by Tersoff ${ }^{4}$ in 1988 , there is still no perfect potential that is suitable for various carbon-based systems. Following the footprint of Tersoff, many researchers have 
developed and improved potential functions of carbon systems such as the reactive empirical bondorder (REBO) potential ${ }^{5}$ developed by Brenner in 1990; the AIREBO potential ${ }^{6}$ in 2000; the first ReaxFF potential ${ }^{7}$ in 2001; etc. The comparative study conducted by Nigel A. Marks has provided a good summary of carbon potentials. ${ }^{8}$ In this work, we tested six typical carbon potentials including Tersoff, AIREBO, BOP, ${ }^{9} \mathrm{Ci}-\operatorname{ReaxFF},{ }^{10} \operatorname{ReaxFF} 2013,{ }^{11}$ and the initial $\operatorname{ReaxFF}{ }^{7}$ to find a suitable potential to simulate the phase transformation from amorphous carbon to nanocrystalline carbon, and the results are shown in Figure S3 and Figure S4. All of the force fields were used as implemented in LAMMPS, with no modifications, and all of the simulations follow the same conditions, i.e., $573 \mathrm{~K}$ and no Ar ion bombardment. For comparisons, we first performed a classic MD simulation using the Ci-ReaxFF and found that a total amorphous carbon structure was obtained (Figure S3a), indicating that the nanocrystalline carbon structures were unable to be simulated by the classic MD simulation because of the limitation of the timescale. Then, we performed a series of hybrid MD/tfMC simulations using the six different carbon potentials, as shown in Figure S3b g. For the potentials Tersoff (Figure S3b) and AIREBO (Figure S3c), even though the tfMC method was coupled to the classic MD, there was no ordered structure. For the potential BOP (Figure S3d), several graphene sheets were observed after the deposition simulations. However, due to the short cut off radius of carbon atoms, i.e., $2.37 \AA$, these graphene sheets were not closely packed into a graphite-like crystal. For the family of ReaxFF potentials, we tested three kinds of potentials. Similar to the results obtained from the BOP potential, the final structure of deposited carbon films simulated by the initial ReaxFF potential (Figure S3g) also 
contained many loose graphene sheets. Actually, the first ReaxFF potential was initially designed for hydrocarbons, which are usually a gas system. Therefore, in 2013, the same group developed a new ReaxFF potential denoted as ReaxFF2013, which is better fitted to describe condensed carbon systems, such as graphite or diamond. The final structure simulated by ReaxFF2013 mainly showed the nanocrystalline structures contained in a vast 3-dimensional graphene networks. For the Ci-ReaxFF potential, the final structure contained more typical nanocrystalline structures such as graphite nanocrystals and graphene nanosheets. Based merely on these results, the force fields Ci-ReaxFF and ReaxFF2013 may be suitable for modeling nanocrystalline carbon films.

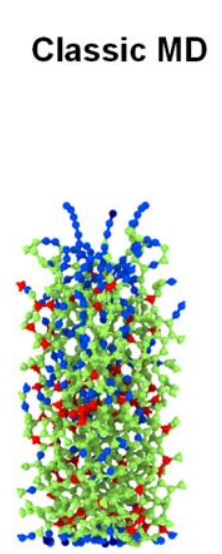

(a) Ci-ReaxFF

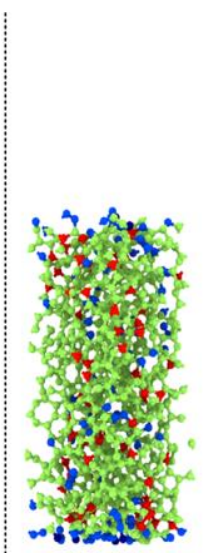

(b) Tersoff

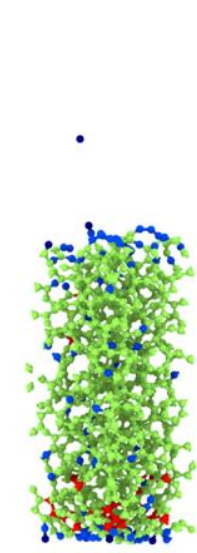

(c) AIREBO
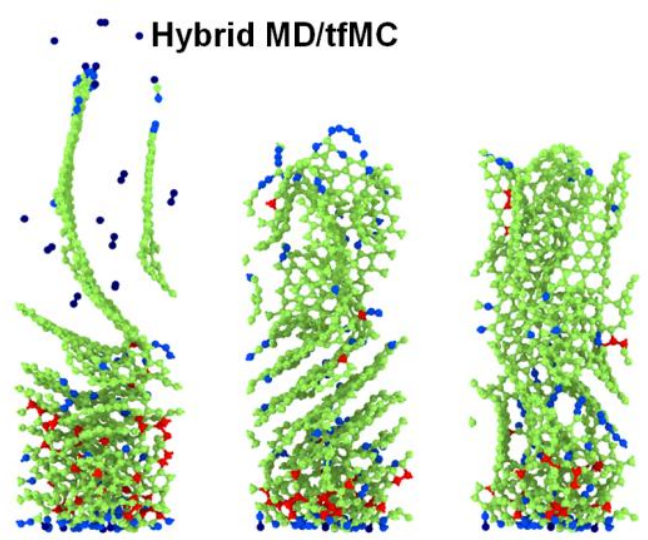

(d) BOP

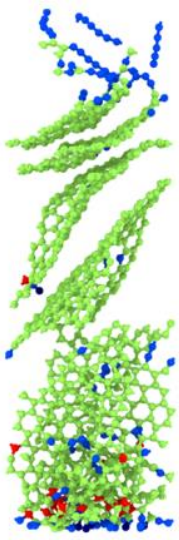

(g) Initial ReaxFF

Figure S3. Simulated carbon films with different potential functions and different techniques. (a)

Ci-ReaxFF and classic MD. (b) Tersoff and hybrid MD/tfMC. (c) AIREBO and hybrid MD/tfMC.

(d) BOP and hybrid MD/tfMC. (e) Ci-ReaxFF and hybrid MD/tfMC. (f) ReaxFF2013 and hybrid

MD/tfMC. (g) ReaxFF and hybrid MD/tfMC. Blue, green, and red spheres correspond to twofold-

, threefold-, and fourfold-coordinated carbon atoms, respectively. It can be seen the Ci-ReaxFF and ReaxFF2013 potentials give the best description of nanocrystalline carbon films. 
The calculation efficiency of a potential function is a key point to complete the simulation of nanocrystalline carbon films in an affordable time. Figure S4 shows the relative calculation efficiency of the six potential functions. To acquire a better description of carbon systems, one often needs to sacrifice the calculation efficiency. According the results in Figure S3, the CiReaxFF and ReaxFF2013 may be two possible candidates for simulating nanocrystalline carbon systems. Considering their difference in calculation efficiencies, we finally chose the Ci-ReaxFF potential to simulate the nanocrystalline carbon films because its calculation efficiency is nearly twice that of the ReaxFF2013 potential.

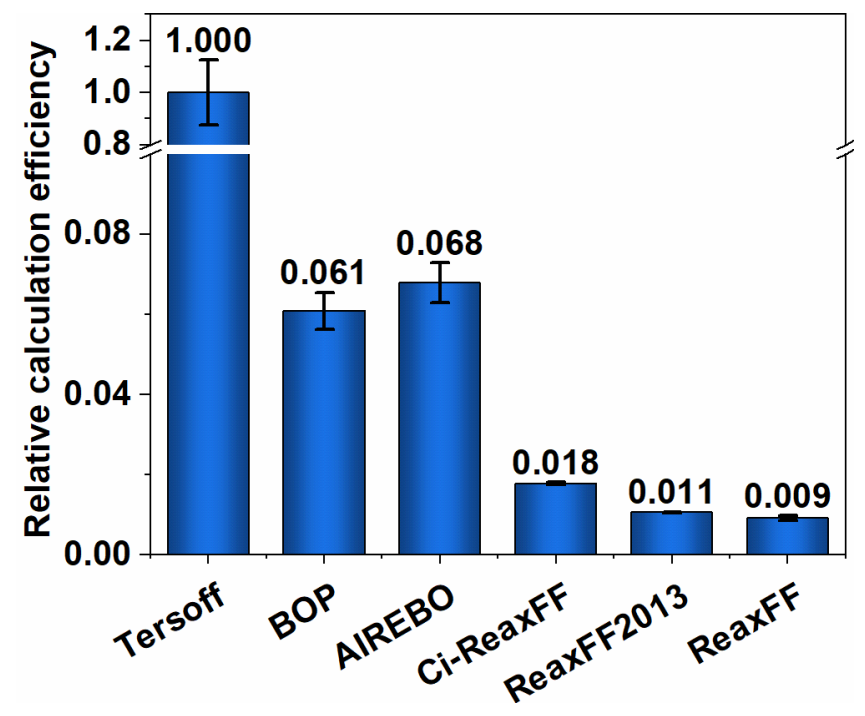

Figure S4. Relative calculation efficiencies of six typical carbon potentials. The number of steps per second of these potentials were all divided by that of the Tersoff potential. In the family of ReaxFF, the Ci-ReaxFF exhibits the highest calculation efficiency. 


\section{S4. Pre-existing stresses induced by different ensembles}

To figure out how much pre-existing tensile stress the NVT and NPT ensemble actually induced, we calculated the time-average stress (every $4 \times 10^{4}$ time steps, namely, $10 \mathrm{ps}$ ) of the graphene using the virial equation and the result is shown in Figure S5. In this calculation, the volume of graphene should first be determined. The width and length of graphene was equal to the corresponding simulation box dimensions, and the thickness of graphene was considered to be the van der Waals diameter of $\mathrm{C}$ atoms, i.e., approximately $3.4 \AA$. As shown in Figure S5, the preexisting stress induced by the NVT ensemble was approximately $2 \mathrm{GPa}$, and the pre-existing stress induced by the NPT ensemble was only approximately $0.3 \mathrm{GPa}$. The pre-existing strain induced by the NVT ensemble makes the graphene break down more easily. Therefore, using the NPT ensemble can help us minimize the effect of the pre-existing strain. However, the graphene-based structures in nanocrystalline carbon films may have a certain pre-existing strain because their boundaries cannot constantly accommodate to the internal tensile stress during the real deposition process. For this reason, the NVT ensemble may be closer to the real situation. Finally, the results obtained from the two ensembles are both included in the Figure 7 of the main manuscript to offer the readers the details about how the pre-existing tensile stress affects the observation of STW defects. 


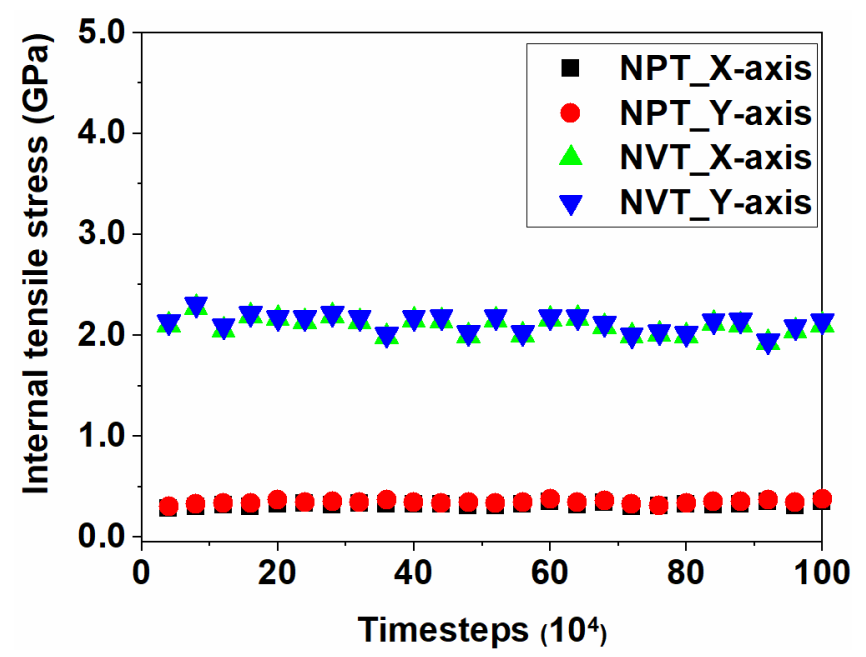

Figure S5. Pre-existing stresses induced by different ensembles at $300 \mathrm{~K}$.

\section{S5. XRD calculation details and the definition of crystallinity}

The XRD intensity was calculated on a mesh of reciprocal lattice nodes defined by the entire simulation domain using a simulated radiation of wavelength $\lambda$, as described by Coleman. ${ }^{12}$ The XRD intensity, $I$, at each reciprocal lattice point, $k$, is computed from the structure factor, $F$, using the equations below:

$$
\begin{gathered}
I=L p(\theta) \frac{F \cdot F}{N} \\
F(\vec{k})=\sum_{j=1}^{N} f_{j}(\theta) \exp \left(2 \pi i \vec{k} \cdot \vec{r}_{j}\right) \\
L p(\theta)=\frac{1+\cos ^{2}(2 \theta)}{\cos (\theta) \sin ^{2}(\theta)} \\
\frac{\sin (\theta)}{\lambda}=\frac{|\vec{k}|}{2}
\end{gathered}
$$

Here, $r j$ is the position of each atom, $f j$ is the atomic scattering factor, $L p$ is the Lorentz-polarization factor, and $\theta$ is the scattering angle of diffraction. In this work, the wavelength was set at 1.5406 
$\AA$ and the scattering angle range was set from $10^{\circ}$ to $60^{\circ}$. Figure S6 presents the results of the calculated XRD intensity of a perfect graphite crystal, and the corresponding crystal faces are also denoted around the main peaks.

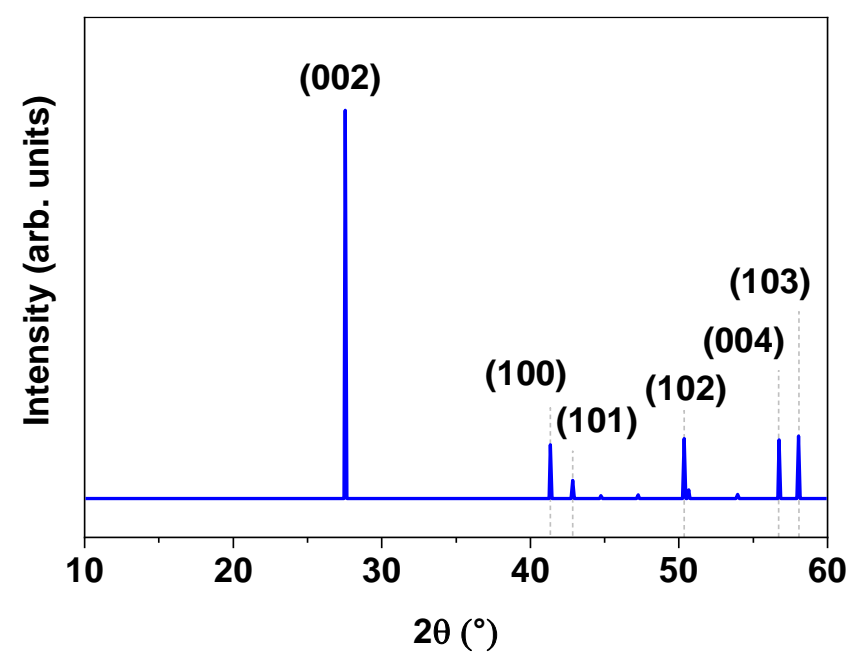

Figure S6. Calculated XRD results of a perfect graphite crystal.

Using the XRD patterns of graphite as a reference, we define the crystallinity of a simulated carbon film as the following equation,

$$
D=\frac{\sum I_{i}^{\text {film }}}{\sum I_{i}^{\text {graphite }}}
$$

which means that the crystallinity was calculated as the ratio between the total XRD intensity of the peaks from nanocrystalline carbon films and perfect graphite crystals. In this work, the three most typical peaks corresponding to the (002), (100), and (101) crystal faces were used for the crystallinity computations. Notably, the distance between (002) crystal faces in nanocrystalline carbon films is often not exactly the same as that in graphite, i.e., $3.4 \AA$, which corresponds to a 
$2 \theta$ of $27^{\circ}$. Therefore, the three most significant peaks at approximately $27^{\circ}$ of nanocrystalline carbon films were all considered to stem from the (002) graphite-like crystal face and their intensities were all included in the crystallinity calculations.

\section{References}

1. Stukowski, A., Visualization and analysis of atomistic simulation data with OVITO-the Open Visualization Tool. Modell. Simul. Mater. Sci. Eng. 2009, 18, 015012.

2. Timonova, M.; Groenewegen, J.; Thijsse, B. J., Modeling diffusion and phase transitions by a uniform-acceptance force-bias Monte Carlo method. Phys. Rev. B 2010, 81, 144107.

3. Mees, M. J.; Pourtois, G.; Neyts, E. C.; Thijsse, B. J.; Stesmans, A., Uniform-acceptance force-bias Monte Carlo method with time scale to study solid-state diffusion. Phys. Rev. B 2012, $85,134301$.

4. Tersoff, J., Empirical Interatomic Potential for Carbon, with Applications to Amorphous Carbon. 1988, 61, 2879-2882.

5. Brenner, D. W., Empirical potential for hydrocarbons for use in simulating the chemical vapor deposition of diamond films. Phys. Rev. B 1990, 42, 9458-9471.

6. Stuart, S. J.; Tutein, A. B.; Harrison, J. A., A reactive potential for hydrocarbons with intermolecular interactions. J. Chem. Phys. 2000, 112, 6472-6486. 
7. van Duin, A. C. T.; Dasgupta, S.; Lorant, F.; Goddard, W. A., ReaxFF: A Reactive Force Field for Hydrocarbons. J. Mater. Chem. A 2001, 105, 9396-9409.

8. De Tomas, C.; Suarez-Martinez, I.; Marks, N. A., Graphitization of amorphous carbons: A comparative study of interatomic potentials. Carbon 2016, 109, 681-693.

9. Zhou, X. W.; Ward, D. K.; Foster, M. E., An analytical bond-order potential for carbon. J. Comput. Chem. 2015, 36, 1719-1735.

10. Kański, M.; Maciążek, D.; Postawa, Z.; Ashraf, C. M.; van Duin, A. C. T.; Garrison, B. J., Development of a Charge-Implicit ReaxFF Potential for Hydrocarbon Systems. J. Phys. Chem. Lett. 2018, 9, 359-363.

11. Srinivasan, S. G.; van Duin, A. C. T.; Ganesh, P., Development of a ReaxFF Potential for Carbon Condensed Phases and Its Application to the Thermal Fragmentation of a Large Fullerene. J. Mater. Chem. A 2015, 119, 571-580.

12. Coleman, S.; Spearot, D.; Capolungo, L., Virtual diffraction analysis of Ni [ [ $\left.\begin{array}{lll}0 & 1 & 0\end{array}\right]$ symmetric tilt grain boundaries. Modell. Simul. Mater. Sci. Eng. 2013, 21, 055020. 